\title{
SYMMETRIC DERIVATES, SCATTERED, AND SEMI-SCATTERED SETS
}

\author{
CHRIS FREILING
}

\begin{abstract}
We call a set right scattered (left scattered) if every nonempty subset contains a point isolated on the right (left). We establish the following monotonicity theorem for the symmetric derivative. If a real function $f$ has a nonnegative lower symmetric derivate on an open interval $I$, then there is a nondecreasing function $g$ such that $f(x)>g(x)$ on a right scattered set and $f(x)<g(x)$ on a left scattered set. Furthermore, if $R$ is any right scattered set and $L$ is any left scattered set disjoint with $R$, then there is a function which is positive on $R$, negative on $L$, zero otherwise, and which has a zero lower symmetric derivate everywhere. We obtain some consequence including an analogue of the Mean Value Theorem and a new proof of an old theorem of Charzynski.
\end{abstract}

\section{INTRODUCTION}

This paper will establish the following theorem, some generalizations, and some consequences. In $\S 2$ we will introduce left scattered, right scattered, and splattered sets and explain their necessary role. In $\S 3$ we will prove a new partition property for symmetric covers. In $\S 4$ we will prove our main results regarding monotonicity. In $\S 5$ we will look at some consequences involving scattered sets, obtaining a symmetric analogue of the Mean Value Theorem and a new proof of a theorem of Charzynski.

Theorem 1.' If a real function $f$ has a nonnegative lower symmetric derivate on some open interval I (i.e., for $x$ in $\left.I, \liminf _{h \rightarrow 0}(f(x+h)-f(x-h)) / 2 h \geq 0\right)$, then there is a countable set $S$ such that $f$ is nondecreasing on $I-S$.

This theorem seems to run contrary to the following example.

Received by the editors July 26, 1988.

1980 Mathematics Subject Classification (1985 Revision). Primary 26A24; Secondary 26A48.

Key words and phrases. Monotonicity, symmetric derivative, scattered sets, symmetric covers, symmetric continuity, symmetric derivation bases.

The author wishes to express thanks to the referee for his insightful comments and especially for introducing right and left scattered sets and indicating their proper role.

1 Note upon revision: A covering property yielding this version of our main result has been independently proved by $\mathrm{D}$. Preiss and B. S. Thomson, A symmetric covering theorem, Real Analysis Exchange 14 (1988-89), 253-254. 
Example 1 (D. Preiss [16] in answer to a question of Hausdorff [10]). There exists a symmetrically continuous function which possesses uncountably many discontinuity points,

Example 2 (Belna, Evans, and Humke [1]). There exists a continuous function $f$ such that if $S=\{x \mid f$ is symmetrically nondecreasing at $x\}$ and $M=\{x \mid f$ is nondecreasing at $x\}$ then $S-M$ is uncountable, where the local property " $f$ is nondecreasing at $x$ " means that $f(x-h) \leq f(x) \leq f(x+h)$ for all $h$ sufficiently close to zero, and " $f$ is symmetrically nondecreasing at $x$ " means that $f(x-h) \leq f(x+h)$ for all $h$ sufficiently close to zero, and

Example (J. Foran, [8]). There exists a continuous function with a finite symmetric derivative everywhere, but which is nondifferentiable at uncountably many points.

We remark that Example 1 has been recently improved upon by M. Chlebik [3], who showed that the collection of everywhere symmetrically continuous functions has power $2^{c}$. Our theorem easily implies that the collection of everywhere symmetrically monotone functions has power only $c$. Also we immediately see that Example 2, if $S$ contains an interval then $S-M$ is countable in that interval.

On the other hand our theorem seems to be strongly suggested by the following theorem which was first proved for countable sets by Sierpiński [18]:

Theorem (Charzynski [2]; cf. Examples 1 and 3). If a function $f$ has finite upper and lower symmetric derivates everywhere (i.e.,

$$
\limsup _{h \rightarrow 0}(f(x+h)-f(x-h)) / 2 h<\infty
$$

and

$$
\left.\liminf _{h \rightarrow 0}(f(x+h)-f(x-h)) / 2 h>-\infty\right)
$$

then the set of discontinuity points of $f$ is scattered.

Here to say that a set is scattered means that every nonempty subset contains an isolated point. A set is scattered iff it is a countable $G_{\delta}$ (see Davies and Galvin [5]). It is also suggested by

Theorem (A. Kintchine [11]). A continuous function with a nonnegative lower symmetric derivate is nondecreasing.

Theorem (Ruzsa [17], see also Davies [4] and Editorial Staff [7]). If a function is symmetrically constant (i.e., $\forall x \exists \delta>0 \forall h \in(0, \delta) f(x+h)=f(x-h))$ then it is constant off a set with countable closure.

In fact, a combination of Khintchine's proof with Charzynski's theorem almost immediately gives the following: 
Theorem (Charzynski and Khintchine). If a function has a finite upper symmetric derivate and a nonnegative lower symmetric derivate everywhere, then it is nondecreasing off some scattered set.

Thus our theorem, although never mentioned, was very nearly proved in the early 1930 s, and seems transparently plausible since an infinite upper symmetric derivate should not cause a function to decrease(?). One might think, therefore, that Charzynski's and Khintchine's results would trivially generalize by eliminating the condition of a finite upper symmetric derivate and replacing "continuous" with "upper semicontinuous," providing an easy proof of our theorem. In Khintchine's case this is easily done. However in Charzynski's proof, the condition of a finite upper symmetric derivate is used in an essential way, even for proving the upper semicontinuous case. The problem might be paraphrased as follows. If $x<y<z$ and $f(x)$ is close to $f(z)$ and $f(y)$ is close to $f(z)$ then one can conclude that $f(x)$ is close to $f(y)$. However if $f(x) \leq f(z)$ and $f(y) \leq f(z)$ then there is nothing which can be deduced about the relationship between $f(x)$ and $f(y)$. One runs into the same problem when trying to copy proofs of similar results by Ruzsa [17] and Davies [4]. In fact, we will show in the next section that Charzynski and Khintchine's theorem does not remain literally true when the finite derivate condition is dropped.

Our results will generalize several other monotonicity theorems for the symmetric derivative, such as

Theorem (M. Evans, [6], see also C. Weil [22]). If $f$ is a measurable function such that

(i) for each $x, \liminf _{a \rightarrow x} f(a) \leq f(x) \leq \limsup _{a \rightarrow x} f(a)$, and

(ii) the lower symmetric derivate of $f$ is nonnegative almost everywhere and greater than negative infinity everywhere,

then $f$ is nondecreasing.

Theorem (L. Larson, [12]). If $f$ is a measurable function whose lower symmetric derivate is nonnegative almost everywhere and greater than negative infinity everywhere, then there is a nondecreasing function $g$ such that $g=f$ on the continuity points of $f$.

There are many other interesting monotonicity theorems for the symmetric derivative, not all of which are immediately implied by our theorem. For further discussion on these and other results in symmetric real analysis we refer the reader to Larson [13].

\section{LEFT SCATTERED, RIGHT SCATTERED, AND SPLATTERED SETS}

We have from Ruzsa's theorems that a symmetrically constant function is constant off some closed countable set. As was observed by Ruzsa, his result is the best possible in the following sense: Every closed countable set is contained in another closed countable set whose characteristic function is symmetrically constant. The reason for this is that "countable" and "closed" are closed under "closure under $2 x-y$ ". 
Charzynski and Khintchine's theorem tells us that a function with a zero symmetric derivative everywhere is constant off some scattered set. The sharpness of this theorem is answered by the following result of Jurek:

Theorem (Jurek, see Szpilrajn [19]). ${ }^{2}$ Let $S$ be any scattered set. Then there is a function which is positive on $S$, zero off $S$, and has a zero symmetric derivative everywhere.

In the next section we will draw a similar connection between nonnegative symmetric derivates and countable sets. In order to classify these countable sets we make the following definitions: A set $A$ is called right scattered (left scattered) iff every nonempty set contains a point isolated on the right (left). $A$ is called splattered iff every nonempty set contains a point isolated on one side. Obviously these are notions of smallness in the sense that any subset of a scattered (resp. right scattered, left scattered, splattered) set is also scattered (resp. right scattered, left scattered, splattered).

Each of these notions is closed under finite unions. For example, let $S$ and $T$ be any two right scattered sets. If some open interval $I$ intersects one of these but not the other, then $S \cup T$ contains a right isolated point in $I$. On the other hand, if $S$ and $T$ are dense in each other and one of them is nonempty, then it contains a right isolated point which is also a right isolated point in their union. Therefore, either $S \cup T$ is empty or it contains a right isolated point. The same holds for any subset of $S \cup T$ and so $S \cup T$ is right scattered.

Any set of countable closure is scattered, any scattered set is right scattered (left scattered), and any right scattered (left scattered) set is splattered. On the other hand, the centers of the complementary intervals of the Cantor set form a scattered set which does not have countable closure, the left (right) endpoints of these same intervals form a right scattered (left scattered) set which is not scattered, and the set of all endpoints of these intervals form a splattered set which is neither right scattered nor left scattered.

Proposition 1. Splattered sets (and hence scattered, right scattered, and left scattered sets) are countable.

Proof. If a set $A$ is uncountable then there is an element $x$ of $A$ such that every closed interval which contains $x$ also contains uncountably many points in $A$. Otherwise for each $x$ in $A$ we assign the largest closed interval $I$ containing $x$, such that $A$ is countable in $I$. Any two intervals thus obtained would be either disjoint or identical. Thus there would be only countably many such intervals and hence only countably many elements in $A$. Therefore if we consider the collection of points $x$ in $A$ with this property we obtain a nonempty subset of $A$ which is bilaterally dense in itself.

Sets of these types can be characterized in terms of their Cantor-Bendixon

${ }^{2}$ A proof appears in Szpilrajn [19]; however in a note appearing in Fund. Math. 22. Szpilrajn acknowledges that it was Jurek who first proved this result. 
derivatives. For any set $A$ and any countable ordinal $\alpha, D^{0}(A)=A, D^{\alpha+1}(A)$ is the set of nonisolated points in $D^{\alpha}(A)$, and if $\alpha$ is a limit ordinal then $D^{\alpha}(A)=\bigcap_{\beta<\alpha} D^{\beta}(A)$. If a set is scattered then when $D^{\alpha}(A)$ is nonempty, $D^{\alpha}(A)-D^{\alpha+1}(A)$ is also nonempty, and therefore for some countable ordinal $\alpha, D^{\alpha}(A)$ must be empty. On the other hand if $D^{\alpha}(A)$ is empty for some ordinal $\alpha$ then for each $x \in A$ there exists an ordinal $\alpha(x)$ such that $x \in$ $D^{\alpha(x)}(A)-D^{\alpha(x)+1}(A)$. Then if $B$ were a nonempty subset of $A$ there would be an $x \in B$ whose corresponding ordinal $\alpha(x)$ is minimal and hence $x$ would be isolated in $B$. Therefore, $A$ is scattered iff for some countable ordinal $\alpha, D^{\alpha}(A)$ is empty.

Similarly we may define

$$
D_{r}^{\alpha}(A)=A, \quad D_{r}^{\alpha+1}(A)=\left\{x \in D_{r}^{\alpha}(A) \mid \forall \varepsilon>0(x, x+\varepsilon) \cap D_{r}^{\alpha}(A) \neq \varnothing\right\},
$$

and for limit $\alpha, D_{r}^{\alpha}(A)=\bigcap_{\beta<\alpha} D^{\beta}(A)$. Then $A$ is right scattered iff for some countable ordinal $\alpha, D_{r}^{\alpha}(A)$ is empty. Similarly we may define $D_{l}^{\alpha}(A)$ so that $A$ is left scattered iff for some countable ordinal $\alpha, D_{l}^{\alpha}(A)$ is empty. Finally let

$$
\begin{aligned}
& D_{S p}^{0}(A)=A, \quad D_{S p}^{\alpha+1}(A)=\left\{x \in D_{S p}^{\alpha}(A) \mid \forall \varepsilon>0(x-\varepsilon, x) \cap D_{S p}^{\alpha}(A) \neq \varnothing\right. \\
&\text { and } \left.(x, x+\varepsilon) \cap D_{S p}^{\alpha}(A) \neq \varnothing\right\},
\end{aligned}
$$

and for limit $\alpha, D_{S p}^{\alpha}(A)=\bigcap_{\beta<\alpha} D_{S p}^{\beta}(A)$. Then $A$ is splattered iff for some countable ordinal $\alpha, D_{S p}^{\alpha}(A)$ is empty.

Proposition 2. $A$ is scattered iff it is the intersection of a right scattered and a left scattered set.

Proof. Let $A$ be scattered. Then $A$ is both left scattered and right scattered and $A=A \cap A$. On the other hand, suppose $A=R \cap L$ where $R$ is right scattered and $L$ is left scattered. Then $A$ is both right scattered and left scattered. Let $C$ be a nonempty subset of $A$. Let $D$ be the set of left isolated points in $C$. Then $D$ is nonempty and, in fact, $D$ must intersect every open interval which intersects $C$. Let $E$ be the set of right isolated points in $D$. Then $E$ is nonempty. But any element in $E$ is isolated in $C$. Therefore $A$ is scattered.

Proposition 3. $A$ is splattered iff it is the union of a right scattered and a left scattered set.

Proof. The union of a right scattered and a left scattered set is also the union of two splattered sets and therefore splattered. On the other hand, suppose $A$ is splattered. For each $x$ in $A$ let $\alpha(x)$ denote the unique ordinal $\alpha$ such that $x \in D_{S p}^{\alpha}(A)-D_{S p}^{\alpha+1}(A)$. Let $R$ denote the set of elements of $A$ which are isolated on the right in $D_{S p}^{\alpha(x)}(A)$, and let $L$ denote the set of elements of $A$ which are isolated on the left in $D_{S p}^{\alpha(x)}(A)$. Clearly $A=R \cup L$. Let $B$ be any nonempty subset of $R$ and let $x$ be an element of $B$ with $\alpha(x)$ minimal. Then 
$x$ is isolated on the right in $D_{S p}^{\alpha(x)}(A)$. Since $\alpha(x)$ is minimal, $B \subset D_{S p}^{\alpha(x)}(A)$ and hence $x$ is isolated on the right from other elements of $B$. Therefore $R$ is right scattered. Similarly $L$ is left scattered.

The following version of Jurek's theorem shows that, in a sense, our forthcoming monotonicity theorem is best possible. It also shows that the finite derivate condition cannot be dropped in Charzynski and Khintchine's theorem:

Theorem 1. Let $R$ be any right scattered set and $L$ be any left scattered set such that $R \cap L$ is empty. Then there is a function $f$ which is positive on $R$, negative on $L$, zero otherwise, and which has a zero lower symmetric derivate everywhere.

Proof. Let $R$ and $L$ be any disjoint sets which are right scattered and left scattered respectively. For $x \in R$, let $\alpha(x)$ denote the unique ordinal $\alpha$ such that $x \in D_{r}^{\alpha}(R)-D_{r}^{\alpha+1}(R)$, and let $\varepsilon(x)$ be such that $(x, x+\varepsilon(x)) \cap D_{r}^{\alpha(x)}(R)$ is empty. Similarly for $x \in L$, let $\alpha(x)$ denote the unique ordinal $\alpha$ such that $x \in D_{l}^{\alpha}(L)-D_{l}^{\alpha+1}(L)$, and let $\varepsilon(x)$ be such that $(x-\varepsilon(x), x) \cap D_{l}^{(\gamma(x)}(L)$ is empty. Let

$$
f(x)= \begin{cases}\varepsilon(x)^{2} & \text { if } x \in R \\ -\varepsilon(x)^{2} & \text { if } x \in L \\ 0 & \text { otherwise }\end{cases}
$$

Since $f$ is zero off a countable set, its lower symmetric derivate is always less than or equal to zero. It remains to show that $f$ has a nonnegative lower symmetric derivate. Fix $x$. Suppose there is some $c \in R$ such that $x \in$ $(c, c+\varepsilon(c))$. Then fix such a $c$ for which $\alpha(c)$ is minimal. Then there are no elements $z<x$ in $R-D_{r}^{\alpha(c)}(R)$ for which $z+\varepsilon(z)>x$. Also there are no elements $z$ in $(c, x)$ which are in $D_{r}^{(r(c)}(R)$. Therefore if $z \in(c, x) \cap R$ then $f(z)=(\varepsilon(z))^{2} \leq(x-z)^{2}$. If there is no such $c \in R$ for which $x \in(c, c+\varepsilon(c))$ then for all $z<x$ in $R$ we have $f(z)=(\varepsilon(z))^{2} \leq(x-z)^{2}$. If $z<x$ is not in $R$ then $f(z) \leq 0$. So in any case there is a number $a<x$ such that for all $z$ in $(a, x), f(z) \leq(x-z)^{2}$.

Similarly, using $L$, there is some $b>x$ such that for all $z$ in $(x, b)$, $f(z) \geq-(z-x)^{2}$. If $h$ is small enough that $(x-h, x+h) \subset(a, b)$ then $f(x+h)-f(x-h) \geq-h^{2}-h^{2}$ and hence $(f(x+h)-f(x-h)) / 2 h \geq-h$ and hence $f$ has a nonnegative lower symmetric derivate.

We will find it convenient to use a simple characterization of scattered, (resp. right scattered, left scattered, splattered) sets in terms of the following games. Since the outcome of each game is settled after a finite number of moves, one of the players must have a winning strategy by a theorem of Gale-Stewart [9].

For each set $A$, we define the two-person perfect information infinite game 
$S p(A)$ as follows:

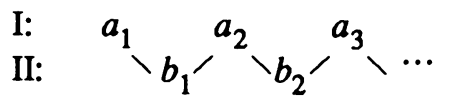

Rules. $a_{i+1}$ and $b_{i+1}$ must be between $a_{i}$ and $b_{i}$. I wins if each $a_{i}$ is in $A$. Suppose we make a slight variation in the game by requiring player II to always play $b_{i}>a_{i}$. Then we call this variation $S c^{+}(A)$. If we instead require that $b_{i}<a_{i}$ then we call it $S c^{-}(A)$. Finally, if we allow player I to decide on which side of each $a_{i}$ player II must play then we call the game $S c(A)$.

Proposition 4. Player II wins $S c(A)$ (resp. $\left.S c^{+}(A), S c^{-}(A), S p(A)\right)$ iff $A$ is scattered (resp. right scattered, left scattered, splattered).

Proof. We prove this for $\mathrm{Sc}^{+}(A)$, the other proofs being similar. If $A$ is not right scattered then $A$ has a nonempty subset $A^{\prime}$ which is dense in itself from the right. Then I will be able to always play an element in $A^{\prime}$. On the other hand, suppose I has a winning strategy. Then let $A^{\prime}$ be the collection of all possible plays for I using this strategy. Then $A^{\prime}$ is nonempty and dense in itself from the right.

It was shown by Davies and Galvin [5] that scattered sets are exactly the sets which are countable and $G_{\delta}$. Analogues for right scattered, left scattered, and splattered also hold.

Proposition 5. $A$ set is splattered (right scattered, left scattered) iff it is both countable and a countable intersection of unions of closed intervals (left closed right open intervals, left open right closed intervals).

Proof. We prove this for splattered sets, the other cases being similar. Suppose $A$ is splattered. We have already shown $A$ is countable. Call a sequence $\left(a_{1}, b_{1}, \ldots, a_{i}, b_{i}\right)$ good for $x$ if it is a sequence of plays in $S p(A)$ where II is following some fixed winning strategy, each $a_{n} \in A$, and $x \in\left(a_{i}, b_{i}\right)$ or $x \in\left(b_{i}, a_{i}\right)$. For each such good sequence, a sequence $\left(a_{1}, b_{1}, \ldots, a_{i+1}, b_{i+1}\right)$ is called better for $x$ if II is still following his winning strategy, each $a_{n} \in A$, and $x \in\left[a_{i+1}, b_{i+1}\right)$ or $x \in\left(b_{i+1}, a_{i+1}\right]$. Let $x \in A^{\prime}$ iff for every good sequence for $x$ there is a better one. Clearly $A^{\prime}$ is a countable intersection of unions of half open intervals and therefore also a countable intersection of unions of closed intervals. Also $A \subset A^{\prime}$. On the other hand, if $x \in A^{\prime}-A$ then every better sequence for $x$ is also a good one, leading to a win for player I. Therefore $A=A^{\prime}$.

In the other direction, suppose $A=\bigcap_{i=1}^{\infty} G_{i}$ where each $G_{i}$ is the union of closed intervals. Suppose also that $A=\left\{x_{1}, x_{2}, \ldots\right\}$ is countable. Assume I has a winning strategy in $S p(A)$. Let II play against this strategy by choosing each $b_{n} \in G_{n}$ so that the sequence $\left(a_{1}, b_{1}, \ldots, a_{n}, b_{n}\right)$ is not good for $x_{n}$ and so that $\lim a_{i}=\lim b_{i}$. Then $\lim b_{i} \in \cap G_{i}-A$ contradicting choice of $A$. 


\section{TRANSITIVE FULL SYMMETRIC COVERS}

In his innovative paper [20], B. Thomson developed a method of covering and partition properties which may be used to generalize many monotonicity results such as the previously mentioned theorems of Larson and Evans. In addition, his method makes no measurability assumptions. As was remarked to me by Michael Evans, the measurability condition in these theorems may also be dispensed with for another reason. The main result in [21] has the following immediate corollary:

Theorem (J. Uher [21]). If a function has a lower symmetric derivate greater than negative infinity on an interval then it is differentiable almost everywhere on that interval (and hence measurable).

The proof of our theorem will depend on this result of Uher to avoid the assumption of measurability, and since we wish to first prove it in the general setting of covering and partition properties, it will also heavily depend upon results and terminology developed by Thomson [20].

Definitions. Let $I$ be an open real interval. Let $S$ be a collection of closed subintervals. We say that $S$ is a symmetric cover (or full symmetric cover) of $I$ if for each $x$ in $I$ there is a number $\delta(x)>0$ such that every interval [ $x-h, x+h]$ with $h<\delta(x)$ belongs to $I$. We say that $S$ is transitive if whenever $[a, b] \in S$ and $[b, c] \in S$ then $[a, c] \in S$.

The following theorem is Thomson's Lemma 3.1 which he attributes to McGrotty [14].

Theorem (McGrotty). Let $S$ be a transitive full symmetric cover of an open interval $I$ and let $x \in I$. Then there is a set $F$ of countable closure such that $S$ contains every subinterval of $I$ whose center is at $x$ and whose endpoints are not in $F$.

In his paper, Thomson derives several monotonicity results from this theorem but states that it is not intended to give a complete picture of the global properties of symmetric covers. As evidence he gives another property of symmetric covers [20, Lemma 3.4]. Our next result shows that the countable set in McGrotty's theorem can be chosen uniformly. (However this still does not completely generalize Thomson's Lemma 3.4 [20].)

Theorem 2. If $S$ is a transitive full symmetric cover of an open interval $I$ then there is a right scattered set $R$ and a left scattered set $L$ such that $\{[a, b] \subset$ $I \mid a \notin R, b \notin L\} \subset S$.

Proof. Let $\alpha$ and $\beta$ be the left and right endpoints of $I$ respectively. Let $T$ be the triangle in $\mathbf{R}^{2}$ with vertices $(\alpha, 0),(\beta, 0)$, and $((\alpha+\beta) / 2,(\beta-\alpha) / 2)$, together with its interior. Let $D$ be the set of points $(x, h)$ in $T$ such that $S$ does not contain the interval $[x-h, x+h]$. For any point $p$ in $T$, we will denote by $x(p)$ and $h(p)$ the first and second coordinates of $p$ respectively, 
and we denote by $l_{m}(p)$ the line segment with slope $m$ which connects $p$ to the $x$-axis. Also, for any two points, $p, q$ in $T$, denote by $p \cdot q$ the intersection point of $l_{1}(p)$ and $l_{-1}(q)$ when this point exists.

Lemma 1. Let $p \in D$ and let $p^{\prime}$ be a point on $l_{1}(p)$ and $p^{\prime \prime}$ be a point on $l_{-1}(p)$ such that $p^{\prime \prime} \cdot p^{\prime}$ is on the $x$-axis. Then at least one of the points $p^{\prime}, p^{\prime \prime}$ must be in $D$.

Proof. Assume both points are not in $D$. Let $p=(x, h), p^{\prime}=\left(x^{\prime}, h^{\prime}\right)$ and $p^{\prime \prime}=\left(x^{\prime \prime}, h^{\prime \prime}\right)$. Then $S$ contains $\left[x^{\prime}-h^{\prime}, x^{\prime}+h^{\prime}\right]$ and $\left[x^{\prime \prime}-h^{\prime \prime}, x^{\prime \prime}+h^{\prime \prime}\right]$. But if $p^{\prime \prime} \cdot p^{\prime}$ lies on the $x$-axis, then it must have an $x$-coordinate of both $x^{\prime}+h^{\prime}$ and $x^{\prime \prime}-h^{\prime \prime}$ and so these must be equal. Then by transitivity, $S$ contains $\left[x^{\prime}-h^{\prime}, x^{\prime \prime}+h^{\prime \prime}\right]$. But $p^{\prime}$ lies on $l_{1}(p)$ and so $x^{\prime}-h^{\prime}=x-h$. Similarly, $x^{\prime \prime}+h^{\prime \prime}=x+h$ contradicting that $(x, h) \in D$.

Lemma 2. Let $p \in D$, and let $\pi_{1}(p)$ and $\pi_{-1}(p)$ be the projections of $D \cap l_{1}(p)$ and $D \cap l_{-1}(p)$ on the $x$-axis respectively. Then at least one of these projections must have measure at least $h(p) / 2$.

Proof. Consider $C_{y}=\{x \mid S$ contains $[y, x]\}$. By the definition of symmetric cover, $(\forall x \in(\alpha, \beta))(\exists \delta(x)>0)(\forall \varepsilon<\delta(x))[x-\varepsilon, x+\varepsilon] \in S$. Hence $(\forall x \in$ $(y, \beta))(\exists \delta(x)>0)(\forall \varepsilon<\delta(x)) x-\varepsilon \in C_{y} \rightarrow x+\varepsilon \in C_{y}$. In other words, the characteristic function of $C_{y}$ has a nonnegative lower symmetric derivate. Therefore by Uher's theorem, $C_{y}$ is measurable. But if $y=x(p)-h(p)$ then $\pi_{1}(p)=\{x \mid(x, x-y) \in D\} \cap[y, x(p)]=\left\{(x+y) / 2 \mid x \notin C_{y}\right\} \cap[y, x(p)]$. Hence $\pi_{1}(p)$ is measurable and, similarly, so is $\pi_{-1}(p)$. Hence, by Lemma 1 , either $\pi_{1}(p)$ has relative measure at least $1 / 2$ in the interval $(x(p)-h(p), x(p))$ or $\pi_{-1}(p)$ has relative measure at least $1 / 2$ in the interval $(x(p), x(p)+h(p))$.

Notation. Let $p \in D$. Then $\pi(p)$ will denote a fixed closed subset, of measure larger than $h(p) / 3$, of either $\pi_{1}(p)$ or $\pi_{-1}(p)$. We let $D_{1}$ denote the collection of $p$ in $D$ for which $\pi(p) \subset \pi_{1}(p)$ and similarly for $D_{-1}$.

Lemma 3. Let $p$ be a point in $T$ and let $A \subset l_{-1}(p)$ and $B \subset l_{1}(p)$ both have uncountable closure. Suppose that for each $p_{1} \in A$ and $p_{2} \in B, p_{1} \cdot p_{2}$ exists and is in $D$. Then there exists an $x$ such that $\{h \mid(x, h) \in D\}$ has uncountable closure.

Proof. We may assume without loss of generality that $A$ and $B$ are both countable, and that $h(A \cdot B)=\inf \left\{h\left(p_{1} \cdot p_{2}\right) \mid p_{1} \in A, p_{2} \in B\right\}$ is greater than zero. Let $A^{\prime}=\left\{p_{1} \in A \mid\right.$ for some $p_{2}$ in $\left.B, p_{1} \cdot p_{2} \in D_{1}\right\}$ and let $B^{\prime}=\left\{p_{2} \in B \mid\right.$ for some $p_{1}$ in $\left.A, p_{1} \cdot p_{2} \in D_{-1}\right\}$. If $p_{1} \in A-A^{\prime}$ then $B^{\prime}$ is equal to all of $B$. Hence either $A=A^{\prime}$ or $B=B^{\prime}$ and we assume without loss of generality that $A=A^{\prime}$. Let $P$ be a nonempty perfect subset of $\operatorname{cl}(A)$. Then $P$ is homeomorphic to a set of positive measure. Induce a measure on $P$ via this homeomorphism.

Let $U$ be $\{(a, c) \mid a \in P$ and for every rational neighborhood $N$ of $a$, there is an $a^{\prime} \in N \cap A$ and $b^{\prime} \in B$ such that $a^{\prime} \cdot b^{\prime} \in D_{1}$ and $\left.c \in \pi\left(a^{\prime} \cdot b^{\prime}\right)\right\}$. It is easy to see that $U$ is an $F_{\sigma \delta}$ subset in the space $P \times \mathbf{R}$. 
Now for each rational neighborhood $N$ of $a \in P$, there is an $a^{\prime} \in A \cap N$, and thus $\left\{c \mid\right.$ for some $a^{\prime} \in N \cap A$ and $b^{\prime} \in B, a^{\prime} \cdot b^{\prime} \in D_{1}$ and $\left.c \in \pi\left(a^{\prime} \cdot b^{\prime}\right)\right\}$ has measure larger than $h(A \cdot B) / 3$. Hence, $\{c \mid(a, c) \in U\}$ has measure at least $h(A \cdot B) / 3$, since it is a nested intersection of sets with measure larger than $h(A \cdot B) / 3$. Therefore by Fubini's theorem, there is some $c$ such that $\{a \mid(a, c) \in U\}$ has positive measure in the induced measure on $P$, and hence is uncountable. But

$$
\begin{aligned}
\{a \mid(a, c) \in U\} & \subset \operatorname{cl}\left\{a^{\prime} \in A \mid\left(\exists b^{\prime} \in B\right) c \in \pi_{1}\left(a^{\prime} \cdot b^{\prime}\right)\right\} \\
& =\operatorname{cl}\left\{a^{\prime} \in A \mid\left(\exists b^{\prime} \in B\right)(\exists h)(c, h) \in D \cap l_{1}\left(a^{\prime} \cdot b^{\prime}\right)\right\} \\
& \subset \operatorname{cl}\left\{a^{\prime} \in l_{-1}(p) \mid(\exists h)(c, h) \in D \cap l_{1}\left(a^{\prime}\right)\right\},
\end{aligned}
$$

and so $\{h \mid(c, h) \in D\}$ also has uncountable closure.

Lemma 4. Let $p \in D_{1}$. Then there is a $p^{\prime} \in l_{1}(p)$ such that $p^{\prime}$ is not on the $x$-axis and $D$ is co-countable in $l_{1}\left(p^{\prime}\right)$. Similarly for $p \in D_{-1}$.

Proof. Since $p \in D_{1}$ we have $D \cap l_{1}(p)$ is uncountable. Let $p^{\prime}$ be a point on $l_{1}(p)$ not on the $x$-axis such that $B=D \cap l_{1}(p)-l_{1}\left(p^{\prime}\right)$ is uncountable. Assume towards a contradiction that $l_{1}\left(p^{\prime}\right)-D$ is uncountable. Let $A=\left\{a \in l_{-1}(p) \mid\right.$ for some $q \in l_{1}\left(p^{\prime}\right)-D, a \cdot q$ is on the $x$-axis $\}$. For every point $q \in l_{1}\left(p^{\prime}\right)-D$ there is a unique such $a \in l_{-1}(p)$ and so $A$ is uncountable. Let $a \in A$ and $b \in B$ and let $q \in l_{1}\left(p^{\prime}\right)-D$ be such that $a \cdot q$ is on the $x$-axis. Then $(a \cdot b) \cdot q$ is also on the $x$-axis. But $a \cdot b \in l_{-1}(b)$ and $b \in D$ and $q \notin D$ and so by Lemma $1, a \cdot b$ must be in $D$. But then by Lemma 3 , there is some $x$ such that $\{h \mid(x, h) \in D\}$ has uncountable closure, but this contradicts McGrotty's theorem [14].

Notation. Let $R=\left\{x(p)-h(p) \mid p \in D_{1}\right\}$ and $L=\left\{x(p)+h(p) \mid p \in D_{-1}\right\}$.

Lemma 5. $R$ is right scattered.

Proof. We describe a winning strategy for player II in the game $\mathrm{Sc}^{+}(R)$. Assuming $a_{i}$ is in $R$, let II play each $b_{i}>a_{i}$ and so close to $a_{i}$ that:

(1) Only countably many $c$ in the interval $\left(a_{i}, b_{i}\right)$ have the property that $\left(c, c-a_{i}\right) \notin D$. This is possible by Lemma 4 since $a_{i} \in R$. We will call this countable exceptional set $E_{i}$.

(2) $b_{i}$ is less than each of the first $i$ points which are greater than $a_{i}$ in each $E_{n}$ where $n \leq i$. Since for each $a_{i}$ there are only finitely many such points to avoid, this will be possible.

(3) $\lim b_{i}=\lim a_{i}$.

Suppose II plays this strategy and I is able to play each $a_{i}$ in $R$. Let $b=\lim b_{i}$. Then $b \notin E_{i}$ for any $i$, and so $\left(b, b-a_{i}\right) \in D$ for each $a_{i}$. But since $\lim a_{i}=b$, we have $(b, h) \in D$ for $h$ arbitrarily close to zero contradicting the definition of symmetric cover. Therefore II has a winning strategy and so $R$ is right scattered. 
Similarly, $L$ is left scattered. Since $D=D_{1} \cup D_{-1}$ we have that for any $(x, h) \in D$, either $x-h \in R$ or $x+h \in L$. In other words if $[a, b] \notin S$ then either $a \in R$ or $b \in L$. This finishes the proof of the theorem.

\section{MONOTONICITY FOR THE LOWER SYMMETRIC DERIVATE}

We are now ready to prove the theorem quoted in the introduction. However in order to make the theorem more applicable with less duplication, we will prove it in a more general setting of interval functions rather than functions of real numbers.

Definitions. We say that $F$ is an interval function on an open interval $I$ if $F$ maps the closed real subintervals of $I$ to real numbers. We let $I^{x}$ denote an interval with center at $x$. We define the upper symmetric derivate $\bar{F}^{s y}(x)$ to be $\lim \sup _{\left|I^{x}\right| \rightarrow 0} F\left(I^{x}\right) /\left|I^{x}\right|$. The lower symmetric derivate $\underline{F}^{s y}(x)$ is defined similarly using lim inf. If the upper and lower symmetric derivates agree then we say that $F$ is symmetrically differentiable at $x$ and call their common value the symmetric derivative of $F$ at $x$ which is denoted by $F^{s y}(x)$. We say that $F$ is symmetrically upper semicontinuous at $x$ iff $\liminf _{\left|I^{x}\right| \rightarrow 0} F\left(I^{x}\right) \geq 0$, and symmetrically lower semicontinuous at $x$ iff $\lim _{\sup _{\left|I^{x}\right| \rightarrow 0}} F\left(I^{x}\right) \leq 0$. If $F$ is both symmetrically upper and lower semicontinuous at $x$ then we say that $F$ is symmetrically continuous at $x$, in which case we have that $\lim _{\left|I^{x}\right| \rightarrow 0} F\left(I^{x}\right)=0$.

There will be three additivity conditions we will be concerned with for interval functions on an open interval $I$. We say that $F$ satisfies the following conditions:

(A) if whenever $a<b<c$ are in $I$ then $F([a, c]) \geq F([a, b])+F([b, c])$,

(B) if whenever $a<b<c$ are in $I$ then $F([a, b]) \geq F([a, c])-|F([b, c])|$, and

(C) if whenever $a<b<c$ are in $I$ then $F([b, c]) \geq F([a, c])-|F([a, b])|$. Theorem 3. If $F$ is an interval function satisfying condition (A) on an open interval I such that:

(1) its lower symmetric derivate is nonnegative almost everywhere,

(2) is greater than negative infinity everywhere except possibly on some countable set, where we at least have that

(3) $F$ is symmetrically upper semicontinuous, then there is a right scattered set $R$ and a left scattered set $L$ such that $F([a, b])$ $\geq 0$ whenever $a \notin R$ and $b \notin L$.

Proof. Let $F$ be as stated and let $A, B$, and $C$ be disjoint sets where $A \cup$ $B \cup C=I$, and (1) is satisfied at all points in $A$ (which has full measure), (2) is satisfied at all points in $B$, and $C$ is a countable set where (3) is satisfied. For each positive integer $m$ let $S_{m 0}=\{J|F(J) /| J \mid>-1 / m\}$ so that for each $m, S_{m 0}$ symmetrically covers $A$. Let $S_{m 1}=\{J \mid$ for some $i$ the center of $J$ is the $i$ th element of $C$ and $\left.F(J)>-1 /\left(m \cdot 2^{i}\right)\right\}$. Then $S_{m 1}$ symmetrically covers $C$, and any collection $\left\{J_{i}\right\}$ of interiorly disjoint intervals in $S_{m 1}$ has 
$\sum F\left(J_{i}\right)>-1 / m$. For $n>1$, let $S_{m n}=\left\{J \mid J \subset G_{m n}\right.$ and $\left.F(J) /|J|>-n\right\}$ where $G_{m n}$ is an open cover of $\left\{x \mid \underline{F}^{s y}(x)\right.$ is between 0 and $\left.-n\right\}$ with measure less than $1 /\left(m n \cdot 2^{n}\right)$. Then for fixed $m, \bigcup\left\{S_{m n} \mid n>1\right\}$ is a symmetric cover of $B$. Furthermore, for any fixed $m$, if $\left\{J_{i}\right\}$ is a sequence of interiorly disjoint intervals each in some $S_{m n}$ where $n>1$, then $\sum F\left(J_{i}\right)>-1 / m$. Let $S_{m}$ be the transitive closure of $\bigcup S_{m n}$. Then $S_{m}$ is a transitive symmetric cover of $I$. Furthermore, if $\left\{J_{i}\right\}$ is a sequence of interiorly disjoint intervals from $\bigcup S_{m n}$ then $\sum F\left(J_{i}\right)>-1 / m+-1 / m+-(1 / m) \sum\left|J_{i}\right|$. Hence if $J \in \cap S_{i}$ then $F(J) \geq 0$, using condition (A).

For each $S_{i}$ there is a corresponding countable (splattered) set from Theorem 2. Therefore, if we consider the union of these sets we have

(*) There is a countable set $N=\left\{n_{1}, n_{2}, \ldots\right\} \subset I$ such that $F(J) \geq 0$ whenever the endpoints of $J$ are in $I-N$.

We may assume without loss of generality that for each point $x$ in $N$ there is an interval $J$ with one endpoint $x$ and the other endpoint in $I-N$, such that $F(J)<0$. Otherwise we may eliminate any counterexamples $x$ from $N$, one by one, without destroying $(*)$.

Let $R=\{x \mid(\exists d(x) \in I-N)(F([x, d(x)])<0\}$. We describe a winning strategy for II in the game $S c^{+}(R)$. Assuming each $a_{i} \in R$ let II play $b_{i}>a_{i}$ so close to $a_{i}$ that:

(1) $b_{i}<a_{i}-F\left(\left[a_{i}, d\left(a_{i}\right)\right]\right) / 2 i$ (that is, $\left.F\left(\left[a_{i}, d\left(a_{i}\right)\right]\right) /\left(b_{i}-a_{i}\right)<-2 i\right)$. Since $F\left(\left[a_{i}, d\left(a_{i}\right)\right]\right)$ is negative, this is possible.

(2) If $A_{i}=\left\{\left(a_{p}+n_{q}\right) / 2 \mid p \leq i\right.$ and $\left.q \leq i\right\}$ then choose $b_{i} \in I$ less than $x$ for each $x \in A_{i}$ which is greater than $a_{i}$. Since there are only finitely many such $x$, this is possible.

(3) $2 b_{i}-a_{i}<d\left(a_{i}\right)$.

(4) If $C=\left\{c_{1}, c_{2}, \ldots\right\}$ then choose $b_{i}<x$ for each $x \in\left\{c_{1}, \ldots, c_{i}\right\}$ which is greater than $a_{i}$.

(5) $\lim a_{i}=\lim b_{i}$.

Suppose II plays this strategy and I is able to play each $a_{i} \in R$. Let $b=$ $\lim b_{i}$. Then by (2), $b$ is not the midpoint of an interval $\left[a_{p}, n_{q}\right]$ with $n_{q} \in N$. In other words, $2 b-a_{i} \notin N$ for any $i$. Therefore, using (3),

$$
F\left(\left[2 b-a_{i}, d\left(a_{i}\right)\right]\right) \geq 0 .
$$

Then by additivity condition $(\mathrm{A})$ we have that

$$
F\left(\left[a_{i}, 2 b-a_{i}\right]\right) \leq F\left(\left[a_{i}, d\left(a_{i}\right)\right]\right) .
$$

But then (1) gives us that $F\left(\left[a_{i}, 2 b-a_{i}\right]\right) /\left(2\left(b-a_{i}\right)\right)<-i$ and hence $\underline{F}^{s y}(b)=$ $-\infty$. But then $b \in C$ contradicting (4).

Therefore II has a winning strategy in $S c^{+}(R)$ and hence $R$ is right scattered. An identical proof shows $L=\{x \mid(\exists d \in I-N) F([d, x])<0\}$ is left scattered. Now suppose $[a, b] \subset I$ and $F([a, b])<0$. Choose any $d$ in $(a, b)-N$. Then by condition (A), one of $F([a, d]), F([d, b])$ is less than zero. Hence either $a \in R$ or $b \in L$. This finishes the proof of the theorem. 
For the case when $F([a, b])=f(b)-f(a)$, which easily satisfies all three additivity conditions, we obtain the following corollary.

Corollary. If $f$ is a real function with a nonnegative lower symmetric derivate, then there is a monotone function $g$ such that $\{x \mid f(x)>g(x)\}$ is right scattered and $\{x \mid f(x)<g(x)\}$ is left scattered.

\section{SOME SCATTERED RESUltS}

Theorem 4. If $F$ is an interval function satisfying additivity conditions (A), (B), and $(\mathrm{C})$ on an open interval I such that

(1) its lower symmetric derivate is nonnegative almost everywhere,

(2) both the upper and lower symmetric derivates are finite, except possibly on some countable set, where we at least have that

(3) $F$ is symmetrically upper semicontinuous, then $F(J) \geq 0$ for all $J$ with endpoints in $I-S$ (where $S$ is scattered). Proof. Since $F$ satisfies the hypothesis of Theorem 3, the proof of Theorem 3 remains valid. Let $G$ be the countable set $\left\{g_{1}, g_{2}, \ldots\right\}$ where one of the symmetric derivates of $F$ takes on an infinite value. We will show under the additional hypothesis that II wins $S c^{-}(R-L)$. Assume $I$ has played each $a_{i} \in R$. Let II play $b_{i}<a_{i}$ so close to $a_{i}$ that:

(1) $b_{i}>a_{i}+\left(F\left(\left[a_{i}, d\left(a_{i}\right)\right]\right)\right) / 2 i$ (i.e., $\left.\left(-F\left(\left[a_{i}, d\left(a_{i}\right)\right]\right)\right) /\left(2\left(a_{i}-b_{i}\right)\right)>i\right)$.

(2) If $A_{i}=\left\{\left(a_{p}+n_{q}\right) / 2 \mid p \leq i\right.$ and $\left.q \leq i\right\}$ then choose $b_{i}>x$ for each $x$ in $A_{i}$ which is less than $a_{i}$. Since there are only finitely many such $x$ this is possible.

(3) $b_{i}>x$ for each $x \in\left\{g_{1}, \ldots, g_{i}\right\}$ which is less than $a_{i}$.

(4) $\lim a_{i}=\lim b_{i}$.

Suppose II plays this strategy and I is able to play each $a_{i}$ in $R-L$. Let $b=$ $\lim b_{i}$. Then by (2), $b$ is not the midpoint of an interval $\left[n_{q}, a_{p}\right]$ with $n_{q} \in N$. In other words, $2 b-a_{i} \notin N$ for any $i$. Hence $F\left(\left[2 b-a_{i}, d\left(a_{i}\right)\right]\right) \geq 0$ since the endpoints are not in $N$. Then by additivity condition (C), $\left|F\left(\left[2 b-a_{i}, a_{i}\right]\right)\right| \geq$ $-F\left(\left[a_{i}, d\left(a_{i}\right)\right]\right)$. Therefore, (1) gives us that $\left|F\left(\left[2 b-a_{i}, a_{i}\right]\right)\right| /\left(2\left(a_{i}-b\right)\right)>i$, and hence $\bar{F}^{s y}(b)=\infty$ or $\underline{F}^{s y}(b)=-\infty$. But then $b \in G$ contradicting (3).

Therefore II has a winning strategy in $\operatorname{Sc}^{-}(R-L)$ and hence $R-L$ is right scattered. Since we showed in Theorem 3 that $R$ is right scattered, $R-L$ must be scattered. Similarly $L-R$ is scattered (using additivity condition (B)). Therefore $N=R \cup L$ is scattered which finishes the proof of the theorem.

The next theorem is reminiscent of the Mean Value Theorem for the ordinary derivative. For a survey of similar theorems see Larson [13].

Theorem 5. If $f$ is a real function with finite symmetric derivates on an open interval $I$ then for all $a<b$ in $I-S$ (where $S$ is scattered) and for all $\varepsilon>0$, there is a sequence of numbers $x_{0}, x_{1}, \ldots, x_{n}$ in $I$ with $x_{0}=a$ and $x_{n}=b$ such that

$$
\sum \underline{f}^{s y}\left(c_{i}\right)\left(x_{i}-x_{i-1}\right)-\varepsilon \leq f(b)-f(a) \leq \sum \bar{f}^{s y}\left(c_{i}\right)\left(x_{i}-x_{i-1}\right)+\varepsilon,
$$


where $c_{i}=\left(x_{i}+x_{i-1}\right) / 2$. If in addition we wish to require that $x_{0}<x_{1}<\cdots<$ $x_{n}$, then $S$ will denote a splattered set.

Proof. Let $F([a, b])=\sup \left\{-\varepsilon \mid \varepsilon>0\right.$ and there is a desired sequence $\left\{x_{i}\right\}$ for $\varepsilon$ on the interval $[a, b]\}$. Since $F \leq 0, \bar{F}^{s y} \leq 0$ on $I$. Also, $\underline{F}^{s y} \geq 0$ on $I$. To see this, let $x \in I$ and suppose $h>0$ is so small that $\underline{f}^{s y}(x)-\varepsilon \leq$ $(f(x+h)-f(x-h)) / 2 h \leq \bar{f}^{s y}(x)+\varepsilon$. Then $2 h \underline{f}^{s y}(x)-2 h \varepsilon \leq f(x+h)$ $-f(x-h) \leq 2 h \bar{f}^{s y}(x)+2 h \varepsilon$. Then $x_{0}=x-h$ and $x_{1}=x+h$ will be a desired sequence for $2 h \varepsilon$ on the interval $[x-h, x+h]$. Then $F([x-h, x+h]) \geq-2 h \varepsilon$. Then $F([x-h, x+h]) / 2 h \geq-\varepsilon$. Thus $\underline{F}^{s y}(x) \geq 0$.

By Theorem 3 or else by Theorem 4 , there is a set $S$ such that $F \geq 0$ for closed intervals with endpoints in $I-S$. Since $F \leq 0$ by definition, we have $F=0$ on $I-S$. The difference lies in the fact that if we only require that $x_{0}, x_{1}, \ldots, x_{n}$ be in $I$ then $F$ is easily seen to satisfy conditions (A), (B), and (C). Whereas if we require that $x_{0}<x_{1}<\cdots<x_{n}$ then $F$ only satisfies (A). Hence in the former case $S$ is scattered by Theorem 4 and in the latter case $S$ is splattered by Theorem 3 . This finishes the proof.

We will now give a new proof of Charzynski's theorem.

Theorem 6. Let $f$ be a real function on an open interval I. Suppose that $f$ has finite symmetric derivates everywhere on this interval (with possibly a countable number of exceptions where we at least have that $f$ is symmetrically continuous). Then the discontinuity points of $f$ are scattered.

Proof. Let $F([a, b])=\sup \{-\varepsilon \mid \varepsilon>0$ and $(\exists \delta>0)(\forall \eta$ with $|\eta|<\delta) f(b+\eta)$ $-f(a-\eta)$ is within $\varepsilon$ of $f(b)-f(a)\}$. We note that if $F([a, b])=0$ then $f$ is continuous at $a$ iff $f$ is continuous at $b$. We also note (using symmetric continuity) that $F$ satisfies additivity conditions (A), (B), and (C).

Lemma 1. If the upper and lower symmetric derivates of $f$ are finite at $x$ then $\underline{F}^{s y}(x) \geq-\left(\bar{f}^{s y}(x)-\underline{f}^{s y}(x)\right)$.

Proof. Fix $x$ and for each $\varepsilon>0$ choose $\delta>0$ so small that $2 h\left(\underline{f}^{s y}(x)-\varepsilon\right)<$ $(f(x+h)-f(x-h))<2 h\left(\bar{f}^{s y}(x)+\varepsilon\right)$ for all $0<h<2 \delta$. Consider the interval $[x-\delta, x+\delta]$ and let $\eta$ be such that $|\eta|<\delta$. Then

$$
\begin{gathered}
2(\delta+\eta)\left(\underline{f}^{s y}(x)-\varepsilon\right)<f(x+\delta+\eta)-f(x-\delta-\eta)<2(\delta+\eta)\left(\bar{f}^{s y}(x)+\varepsilon\right), \\
2 \delta\left(\underline{f}^{s y}(x)-\varepsilon\right)<f(x+\delta)-f(x-\delta)<2 \delta\left(\bar{f}^{s y}(x)+\varepsilon\right)
\end{gathered}
$$

and so $f(x+\delta+\eta)-f(x-\delta-\eta)$ is within

$$
2 \delta\left(\bar{f}^{s y}(x)-\underline{f}^{s y}(x)\right)+2 \eta\left(\left|\bar{f}^{s y}(x)\right|+\left|\underline{f}^{s y}(x)\right|\right)+6 \delta \varepsilon
$$

of $f(x+\delta)-f(x-\delta)$. Therefore,

$$
F([x-\delta, x+\delta]) \geq-2 \delta\left(\bar{f}^{s y}(x)-\underline{f}^{s y}(x)\right)-6 \delta \varepsilon .
$$

Hence $F([x-\delta, x+\delta]) / 2 \delta \geq-\left(\bar{f}^{s y}(x)-\underline{f}^{s y}(x)\right)-3 \varepsilon$. Hence $\underline{F}^{s y}(x) \geq$ $-\left(\bar{f}^{s y}(x)-\underline{f}^{s y}(x)\right)$. 
Lemma 2. If $f$ is symmetrically continuous at $x$ then $F$ is symmetrically upper semicontinuous at $x$.

Proof. Fix $x$ and for each $\varepsilon>0$ choose $\delta$ so small that

$$
(\forall h<2 \delta)|f(x+h)-f(x-h)|<\varepsilon .
$$

Consider $[x-\delta, x+\delta]$. If $\eta<\delta$ then $|f(x+\delta+\eta)-f(x-\delta-\eta)|<\varepsilon$. Therefore, $f(x+\delta+\eta)-f(x-\delta-\eta)$ is within $2 \varepsilon$ of $f(x+\delta)-f(x-$ $\delta)$. Therefore, $F([x-\delta, x+\delta]) \geq-2 \varepsilon$. Hence $F$ is upper symmetrically semicontinuous at $x$.

If $f$ is symmetrically differentiable at $x$ then Lemma 1 tells us that $\underline{F}^{s y}(x) \geq$ 0 . By Uher's theorem ([21, Theorem 1]) this happens almost everywhere. Also since $F(J) \leq 0, \bar{F}^{s y}(x)<\infty$. By Theorem 4 we get that $F(J) \geq 0$ for all closed subintervals $J$ with endpoints in $I-S$ where $S$ is scattered. But $F(J) \leq 0$ by definition. Therefore for all $a, b$ in $I-S, f$ is continuous at $a$ iff $f$ is continuous at $b$. Since $f$ must be continuous at some point in $I-S$ (for example by Uher's theorem), the theorem is proved.

Corollary. If $f$ is a real function with finite symmetric derivates on an open interval $I$ where $\int_{I} \underline{f}^{s y}(x)$ exists (in the sense of Denjoy-Perron). Then for all $a, b$ in $I-S$ (where $S$ is scattered) we have $\int_{a}^{b} \underline{f}^{s y}(x)=f(b)-f(a)$.

Proof. Let $U(x)$ be a super-function for $\underline{f}^{s y}$ (in the sense of Perron; see, for example, [15]). Then $U(x)$ is continuous and $\underline{U}^{s y}(x) \geq \underline{f}^{s y}(x)$ for all $x$ in $I$. By Uher's theorem $f^{\prime}(x)$ exists almost everywhere on $I$, giving that $\bar{f}^{s y}=\underline{f}^{s y}$ a.e. Hence $U(x)-f(x)$ has a lower symmetric derivate which is nonnegative a.e. and greater than negative infinity everywhere. Hence $U(x)-$ $f(x)$ is nondecreasing on the continuity points of $f$ (this fact follows from Larson's theorem mentioned in the introduction, see [12]). Similarly, for any subfunction $L(x), f(x)-L(x)$ is nondecreasing on the continuity points of $f$. Hence if $l$ is the left endpoint of $I$, then $\int_{l}^{x} \underline{f}^{s y}(x)-f(x)$ is constant where $f$ is continuous. Hence, if $f$ is continuous at $a$ and $b$ then $\int_{a}^{b} \underline{f}^{s y}(x)=$ $\int_{l}^{b} \underline{f}^{s y}(x)-\int_{l}^{a} \underline{f}^{s y}(x)=f(b)-f(a)$.

We leave open the following question, which was mentioned in the introduction:

Question. If $f$ has a lower symmetric derivate greater than negative infinity everywhere on an open interval $I$, does it follow that $f$ is upper semicontinuous on $I-S$ where $S$ is countable? (splattered?)

\section{REFERENCES}

1. C. L. Belna, M. J. Evans, and P. D. Humke, Symmetric monotonicity, Acta Math. Acad. Sci. Hungar. 34 (1979), 17-22.

2. Z. Charzynski, Sur les fonctions dont la derivee symetrique est partout finie, Fund. Math. 21 (1933), 214-225. 
3. M. Chlebik, On symmetrically continuous functions, Real Anal. Exchange 13 (1987-88), 34.

4. Roy O. Davies, Symmetric sets are measurable, Real Anal. Exchange 4 (1978-79), 87-89.

5. Roy O. Davies and Fred Galvin, Solution to Query 5, Real Anal. Exchange 2 (1976), 74-75.

6. M. J. Evans, A symmetric condition for monotonicity, Bull. Math. Inst. Acad. Sinica 6 (1978), 85-91.

7. Editorial Staff, Concerning Query 37, Real Anal. Exchange 4 (1978-79), 82-83.

8. J. Foran, The symmetric and ordinary derivative, Real Anal. Exchange 2 (1977), 105-108.

9. D. Gale and F. M. Stewart, Infinite games of perfect information, Ann. of Math. Stud., no. 28, Princeton Univ. Press, Princeton, N.J., 1953, pp. 245-266.

10. F. Hausdorff, Problem 62, Fund. Math. 25 (1935), 578.

11. A. Khintchine, Recherches sur la structure des fonctions measurables, Fund. Math. 9 (1927). 212-279.

12. L. Larson, The symmetric derivative, Trans. Amer. Math. Soc. 277 (1983), 589-599.

13. __ Symmetric real analysis: a survey, Real Anal. Exchange 9 (1983-1984), 154-178.

14. J. McGrotty, A theorem on complete sets, J. London Math. Soc. 37 (1962), 338-340.

15. Natanson, Theory of functions of a real variable, Ungar, New York, 1964.

16. D. Preiss, A note on symmetrically continuous functions, Časopis Pěst. Mat. 96 (1971), 262-264.

17. I. Z. Ruzsa, Locally symmetric functions, Real Anal. Exchange 4 (1978-1979), 84-86.

18. W. Sierpiński, Sur une hypothese de M. Mazurkiewicz, Fund. Math. 11 (1928), 148

19. E. Szpilrajn, Remarque sur la derivee symetrique, Fund. Math. 21 (1931), 226-228.

20. B. S. Thomson, On full covering properties, Real Anal. Exchange 6 (1980-81), 77-93.

21. J. Uher, Symmetrically differentiable functions are differentiable almost everywhere, Real Anal. Exchange 8 (1982-83), 253-261.

22. C. E. Weil, Monotonicity, convexity and symmetric derivatives, Trans. Amer. Math. Soc. 222 (1976), 225-237.

Department of Mathematics, California State University, San Bernardino, CaliforNIA 92407 